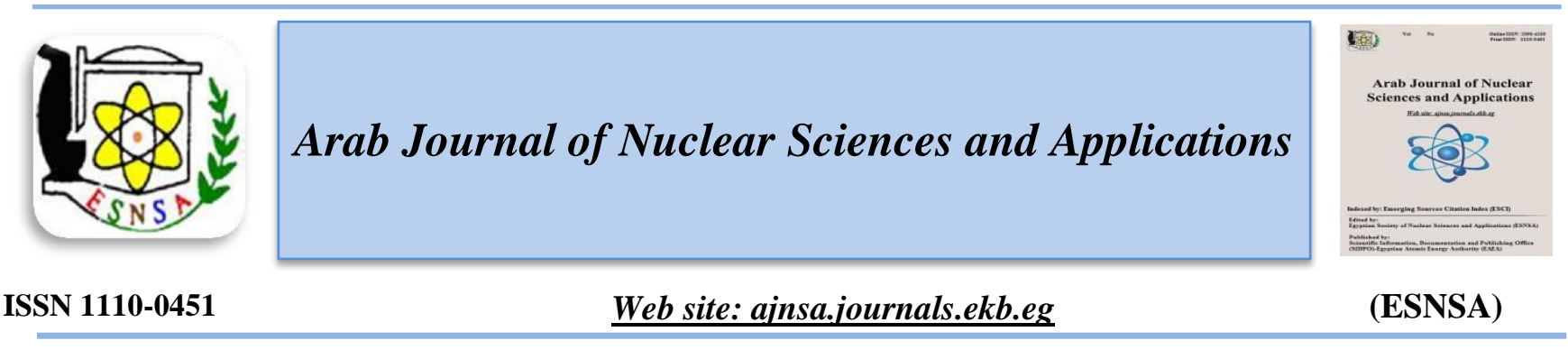

\title{
Developing a New Model for Description of Micro Size Particles in SEM Images for Safeguards Purposes
}

\author{
S. E. Shaban" and M.A. Abdalsamiaa, S. A. El-mongy \\ Nuclear Safeguards and Physical Protection Department, Nuclear and Radiological Regulatory Authority \\ (ENRRA), Cairo, Egypt
}

Received $19^{\text {th }}$ March 2020

Accepted 24 ${ }^{\text {th }}$ Aug. 2020

\begin{abstract}
Scanning electron microscope is a fast screening tool for identification of uranium and thorium particles. In the field of micro-particle analysis for safeguards purposes, estimation of uranium size is necessary especially for scanning of swipes or environmental samples at nuclear facilities. Scanning electron microscope can be used to visualize micro sized particles of any nuclear material. It gives secondary electron images at different magnifications power. In this work, the model JEOL $6510 \mathrm{LV}$ instrument that has capabilities to image samples in a range of magnification from X5 to X300,000 was used. The process of imaging was done at optimum conditions; spot size $=50$, working distance $=10$ and appropriate pressure. The system determines the size of any particle in a secondary electron image using internal image. Many trials were performed to accomplish a correlation between micro size and pixels to estimate the size of targeted particles. In the present study, a mathematical model was developed to describe the relation between micro size and pixels depending on an assumption called "numbers to zeros" assumption. The mathematical description was manipulated in three different ways; linearly, exponentially and by using artificial neural network. The model in its three forms was validated using large numbers of secondary electrons images and comparisons with commercial softwares. The fitting correlation coefficient for linear and exponential manipulation was found to be 0.99997 and 1 respectively.
\end{abstract}

Keywords: Micro-particles; images; Mathematical model; Artificial neural network

\section{Introduction}

Surfaces and components of any investigated target can be observed by Scanning Electron Microscope (SEM). In SEM, a fine beam of electron (called an electron probe) irradiates the specimen leading to generation of secondary electrons (SEs) from the surface of the target. The image resulted from SEs is called SE image. SEIs can give the microscopist information about the topography of the surface [1]. As a matter of fact, SEM is a low cost and rapid technique to image nuclear material samples. Secondary electron images (SEIs) can reveal characteristic information concerning identification and number of $\mathrm{U}$ and $\mathrm{Th}$ in the particles. SEIs can be scanned at different magnification values hence the size of any particle in the image can be easily measured.

\section{Construction of SEM}

The SEM is composed of certain sophisticated components such as optical system to get an electron probe, a stage to put the sample, a SE detector for processing secondary electrons, an image viewing system, and an operation system to achieve various operations [2]. The electron optical system consists of electron gun, a condenser and objective lens to produce an electron probe, a

Corresponding author: smh_elsaid@yahoo.com

DOI: 10.21608/ajnsa.2020.26198.1340

CScientific Information, Documentation and Publishing Office (SIDPO)-EAEA 
scanning coil to scan the electron probe, and other components. To operate SEM ideally without any disturbances to the electron, an optical system evacuation is needed. [3]. Figure (1) shows the main components of SEM.

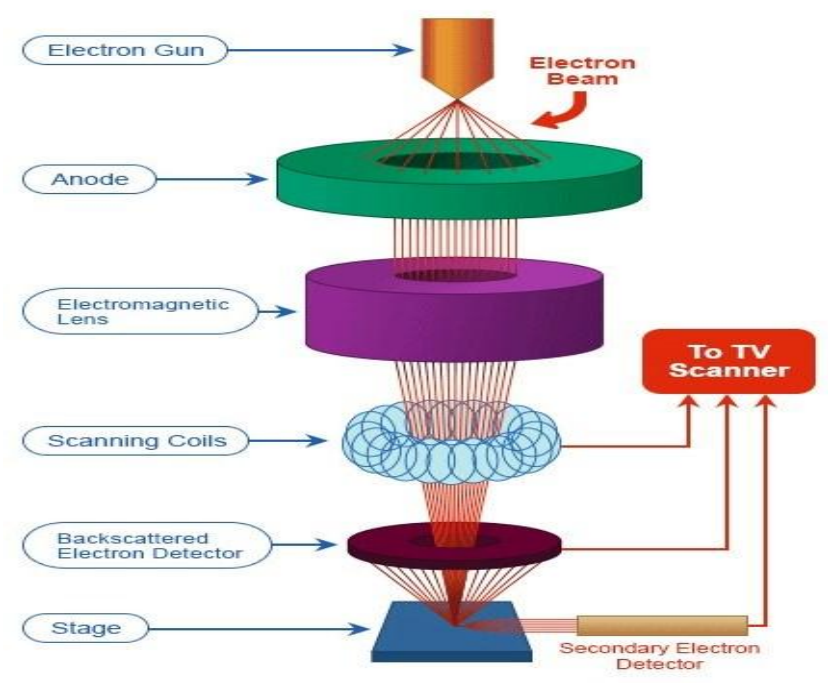

Fig. (1): Components of SEM

Incident electron beam emerges from the electron gun. Electrons are induced by a filament (cathode) through heating at a high temperature. The filament may contain tungsten or may be $\mathrm{LaB}_{6}$. These thermal electrons are collected to form an electron beam, passing to the metal plate (anode) by application of a positive voltage. If a hole is existed at the middle of the anode, the electron beam flows through this pass. Adjustment of the current of the electron beam is performed by Wehnelt electrode that lies in the distance among the cathode and the anode. Applying a voltage to this electrode supports the adjustment process. Thermionic electron gun is the most widely used in SEM instruments. Other electron guns are the field-emission electron gun (FE gun) or the Schottky-emission electron gun (SE gun) [4]. The thermionic emission gun is one of the best and most effective electron sources. The primary components are composed of a heated filament acting as a cathode, a "grid" and an anode. A Schottky emission electron gun is based on fieldassisted thermionic emission. FE gun can lead to acquiring images of a high brightness and contrast.

\section{Image Display and Recording}

Detection of SEs is achieved using SE detector that collect SE emission as a result of bombardment of the sample with primary beam of electron. The outcome signals from the SE detector are amplified and then directed to the viewing unit. Since the scanning on the display unit is synchronized with the electron-probe scan, brightness variation appears on the screen of the display unit, thus forming a SEM image. A cathode-ray tube (CRT) was used for many years as a display unit; however, nowadays, a liquid-crystal display (LCD) has been widely used. In general, the scan speed of the electron probe can be controlled in a number of steps, a fast scan is appropriate for observation while a slow speed is suitable for image acquisition or storing [5].

To save SEM image, in the past, the SEM image appearing on the CRT was photographed with a camera. However, it is now available to record the image in a digital form. This is due to the difficulty to obtain a high-resolution CRT and there are many advantages of electronic file. Hence, it is easier to process images and convenient to send or get image information [5]. SEM images can be acquired at various magnifications. By changing the scan width of the electron probe, the SEM image magnification is also changed.

\section{SEM Role in Nuclear Safeguards}

Nuclear safeguards are a strong tool in the field of non-proliferation [6]. To achieve it in a right manner, certain international requirements should be fulfilled [7]. One of the effective instruments in this field and also in nuclear forensics is SEM. It is used separately or coupled with other instruments to get information about the nuclear materials [8]. This workgets benefit from the imaging system of SEM and tries to conclude a collective relation to estimate the size of any particle located in the image at a certain magnification. The relation is handled mathematically using linear, exponential fitting and Artificial Neural Network (ANN). The resulted model is approved by a comparison with validated software [9].

Artificial neural networks are one of the major tools used in machine learning. As the "neural" part of their name suggests, they are brain-inspired systems which are intended to replicate the way that we humans learn.

\section{Artificial Neural Network}

Neural networks consist of input and output layers, as well as a hidden layer consisting of units that transform the input into something that the output layer can use. They are excellent tools for finding patterns which are far too complex or numerous for a human programmer to extract and teach the 
machine to recognize. An ANN is based on a collection of connected units or nodes called artificial neurons, which loosely model the neurons in a biological brain. Each connection such asthe synapses in a biological brain, can transmit a signal to other neurons. An artificial neuron receives a signal then processes it and enables signal neurons to be connected to it. In ANN, increasing the number of replicates and the value of performance is an indicator of a good prediction.

\section{Experimental work}

The experimental work consists of three ultimate parts. The first part dealt with Scanning images using SEM (JEOL $6510 \mathrm{LV}$ ) and its thermionic electron gun with tungsten filament at working conditions $\mathrm{SS}=50, \mathrm{WD}=10: 12$ and acceleration voltage $=25: 30 \mathrm{keV}$ to get $\mathrm{SEIs}$ at different magnification values. The second part deals mathematically with the data concerning size resulted from SEIs and handling it using different methods. The final part deals with proving the resulted model validity. Before starting such work the SEM micron-bar must be calibrated experimentally using proper Reference Standard sample. This standard may be single element (grid of pure cupper) or an alloy of magnesium and aluminium with specific components. Introducing any of the previous two standards inside sample chamber enable the analyst to magnify the resulted images and the calibration of the micron bar is performed using size estimation software (smile view).

\section{Magnification Notation}

A way of representing the value of any magnification was proposed by authors. This way is based on presenting any magnification value as a number and zeros. Table (1) shows the manner of how to calculate the magnification notation. This Table starts from magnification value X10 to X90.This method can be applied to the SEM magnification range from X10: X300000.

Table (1) represents the first step in building a model to describe the size of any object within SEM image. The second step is concluding a relation between magnification notation and other size parameter.
Calculation of $Y$ Parameter

The value of magnification notation for the range of studied magnifications was plotted versus the ratio between pixel to micro size. The data was fitted with two different methods. Figures (2-9) present data that exponentially fitted while Figure (10) presents the linear fitting between magnification value without zeros and the ratio between pixel and micro size. The pixel size was measured by Matlab. The image J software can also measure the pixel size. The coefficient for linear fitting is 0.99997.

In Figure (9) the value of magnification ranges from 1 to 9 for all studied magnifications and the value of $\mathrm{Y}$ parameter is represented by one linear equation. The value of $\mathrm{Y}$ parameter differs from certain set of magnification to another. The artificial neural network in matlab was used to train data using linear design and the performance at 10000 epoch was $6^{*} 10^{-6}$. Finally, all results obtained from linear, exponential fitting and predictions using ANN were subjected to test against SEMMICRO001 software.

Table (1): The representation of magnification values

\begin{tabular}{cccc}
$\begin{array}{c}\text { Magnification } \\
\text { Value }\end{array}$ & Number & Zeros & $\begin{array}{c}\text { Magnification } \\
\text { Notation }\end{array}$ \\
\hline X 10 & 1 & 1 & 11 \\
X 20 & 2 & 1 & 21 \\
X 30 & 3 & 1 & 31 \\
X 40 & 4 & 1 & 41 \\
X 50 & 5 & 1 & 51 \\
X 60 & 6 & 1 & 61 \\
X 70 & 7 & 1 & 71 \\
X 80 & 8 & 1 & 81 \\
X 90 & 9 & 1 & 91 \\
\hline
\end{tabular}




\section{Results and Discussion}

\section{SEM Analysis}

Figures (11-14) show a sample of SEIs of two different specimens at the same operating conditions with different magnification values. Figures (11,13 and 14) show a metallic sample while figure (12) shows a swipe sample. The swipe sample is simulated using certain type of tissue and it is taken from a glove box that is used to prepare samples for SEM and EDX analysis. The samples are located in location outside facilities (ETZ) at KMP I.

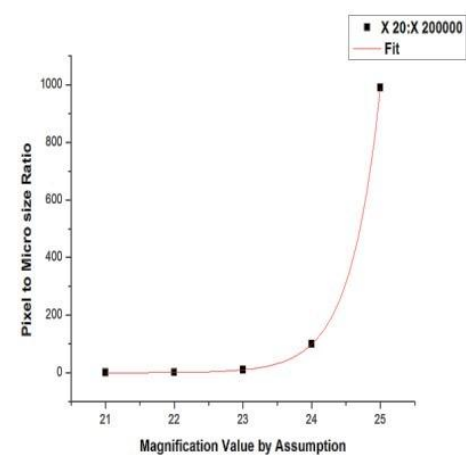

Fig. (2): Exponential fitting for $x 20$ to $\mathbf{x} 200000$

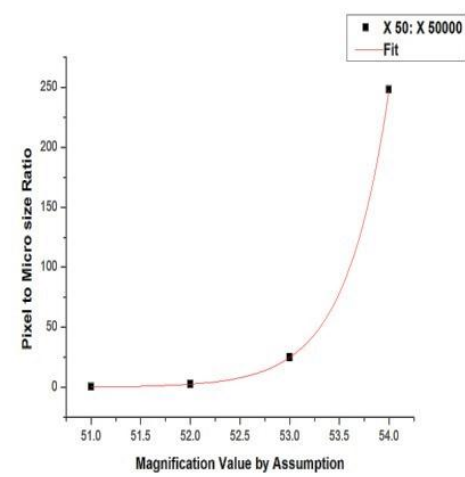

Fig.(5): Exponential fitting for $x 50$ to x 50000

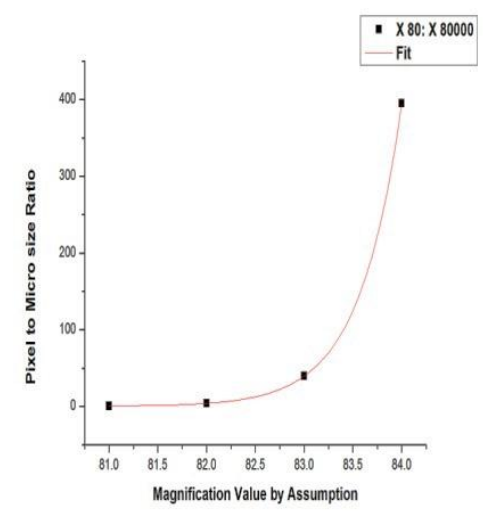

Fig. (8): Exponential fitting for $x 80$ to $\mathrm{x} 80000$

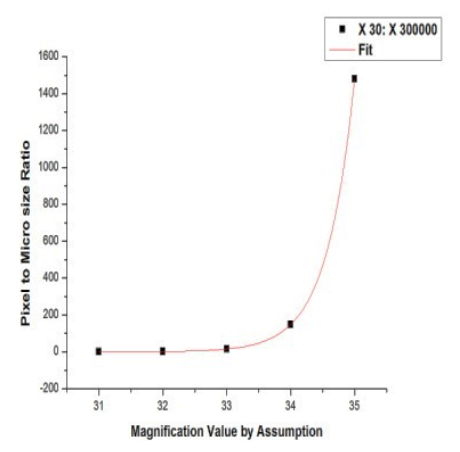

Fig.(3): Exponential fitting for $x 30$ to x 300000

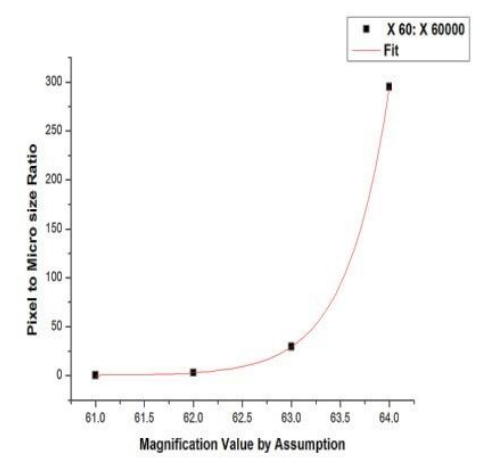

Fig.(6): Exponential fitting for $x 60$ to x 60000

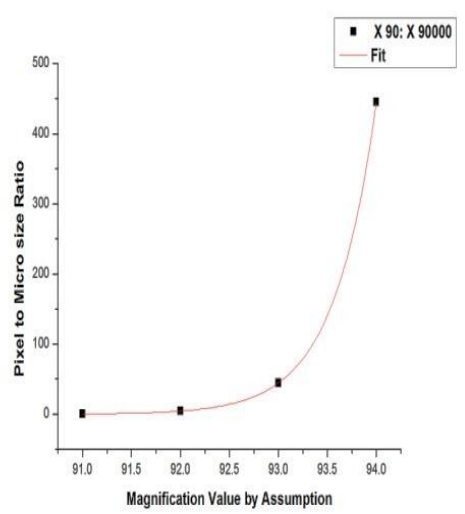

Fig. (9): Exponential fitting for $\mathrm{x} 90$ to x 90000

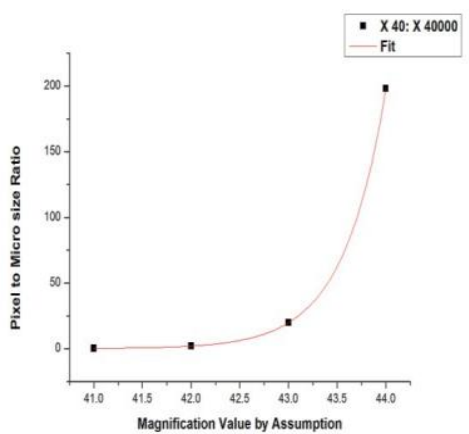

Fig.(4): Exponential fitting for $\mathrm{x} 40$ to $\mathrm{x} 40000$

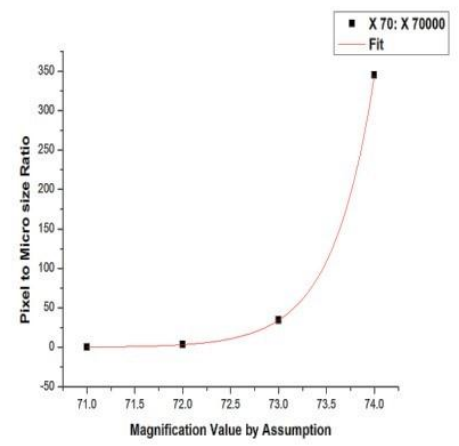

Fig.(7): Exponential fitting for $x 70$ to x 70000

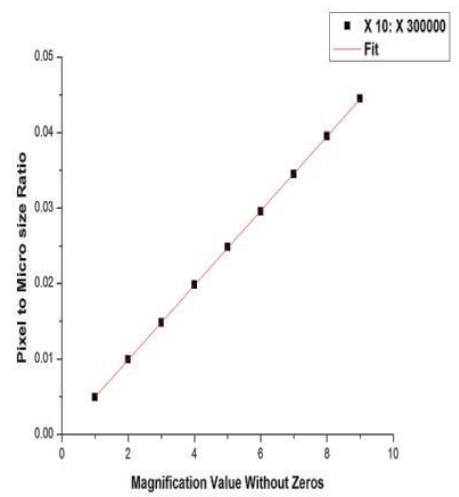

Fig. (10): linear fitting for $\mathrm{x} 10$ to $\mathrm{x}$ 300000 


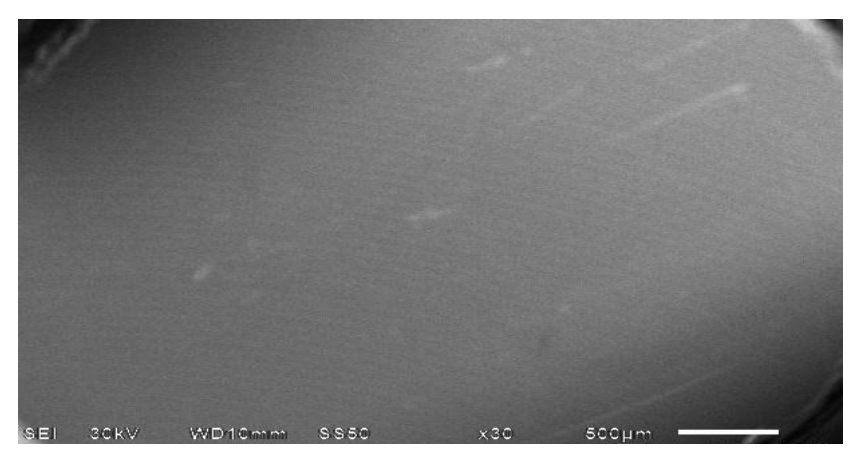

Fig. (11): SEM image at X500

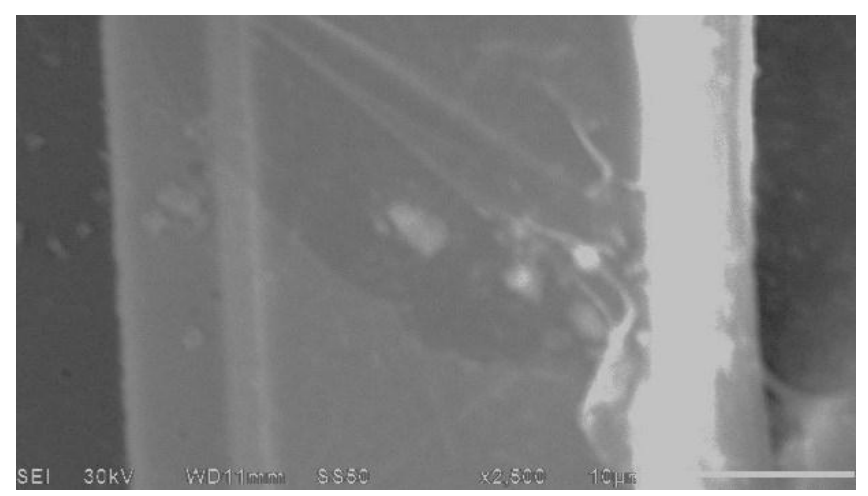

Fig. (13): SEM image at X3000

\section{The Developed Method}

A new mathematical model is used to measure the distance between any two points in SEM images in micro scale by the aid of pixel values. It depends on calculating a parameter called (Y) from the equation resulted from fitting. The fitted equation exists in two forms, the first form is linear while the second form is exponential. The fitted equation was obtained by the previous manipulation of pixel to micro size ratio.

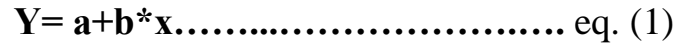

$$
\begin{aligned}
& \mathbf{Y}=\exp \left(\mathbf{a}+\mathbf{b}^{*} \mathbf{x}+\mathbf{c}^{*} \mathbf{x}^{2}\right) \ldots . . . . . . . . . . . . \text { eq. (2) }
\end{aligned}
$$

Where $\mathrm{Y}$ is a calculated parameter, $\mathrm{X}$ is a value related to magnification, while $\mathrm{a}, \mathrm{b}$ and $\mathrm{c}$ are constants that differ in value depending on the type of fitting and the fitted data.

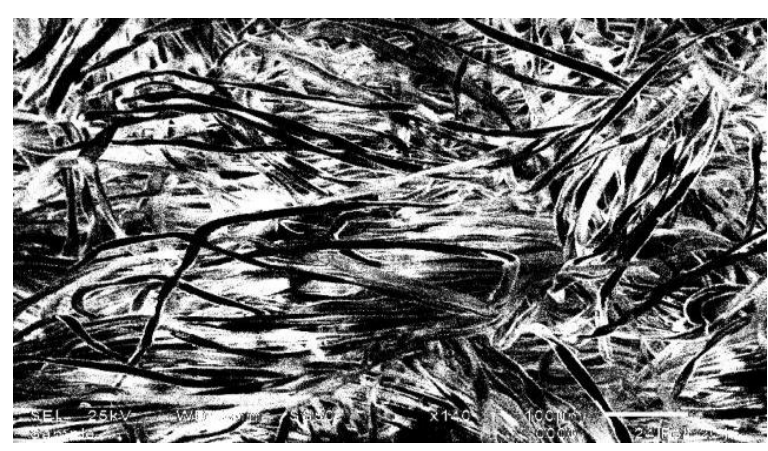

Fig. (12): SEM image at X80

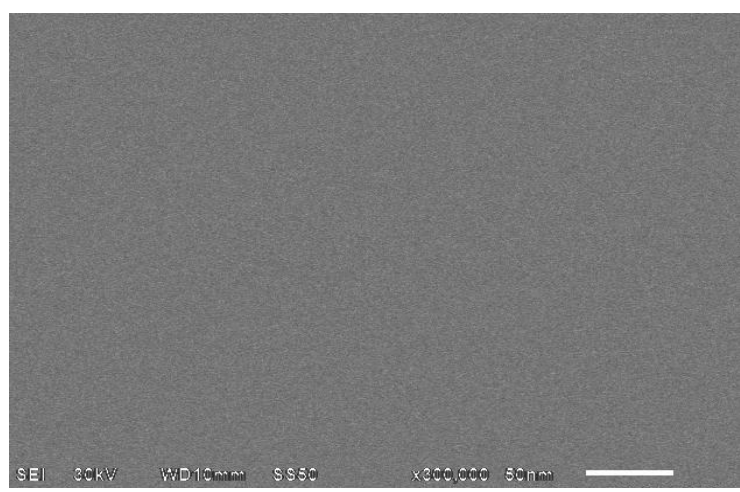

Fig. (14): SEM image at X300000

\section{Model Validation}

The results of micro size were obtained at magnifications from X30 to X300000 using the linear, exponential and ANN methods. These results were compared with that outcome from SEMMicro001 software. Tables (2-6) show the micro size comparison for the abovementioned magnification

Table (2) shows magnification values from X30 to $\mathrm{X} 90$. The exponential fitting method is more accurate than linear fitting and ANN. It is clear that the accuracy of the results increases by going high in the value of magnification

Table (3) shows magnification values from X100 to X900. The exponential fitting method is still more accurate than the linear fitting and ANN. It is clear that the accuracy of results increases by moving high in the value of magnification. The 
most accurate result for all magnifications is that of X900.

Table (4) shows magnification values from X1000 to $\mathrm{X} 9000$. The exponential fitting method is still more accurate than linear fitting and ANN. It is clear that the accuracy of results increases by moving high in the value of magnification. The most accurate result for all magnifications is that of X8000 and X9000 and this behavior appears in linear and exponential fitting.

Table (2): A comparison between linear, exponential and ANN model with valid software at magnifications from X 30 to $X 90$

\begin{tabular}{ccccc}
\hline $\begin{array}{c}\text { Magnification } \\
\text { Value }\end{array}$ & $\begin{array}{c}\text { Linear } \\
\text { Method }\end{array}$ & $\begin{array}{c}\text { Exponential } \\
\text { method }\end{array}$ & ANN & SEMMicro001 \\
\hline X 30 & 499.33 & 499.93 & 496.54 & 500 \\
X 40 & 501.02 & 499.90 & 498.16 & 500 \\
X 50 & 502.03 & 499.88 & 499.13 & 500 \\
X 60 & 199.06 & 199.94 & 197.90 & 200 \\
X 70 & 199.54 & 199.93 & 198.37 & 200 \\
X 80 & 199.90 & 199.92 & 198.73 & 200 \\
X 90 & 200.18 & 199.91 & 199.00 & 200 \\
\hline
\end{tabular}

Table (3): A comparison between linear, exponential and ANN model with valid software at magnifications from $\mathrm{X}$ 100 to $X 900$

\begin{tabular}{ccccc}
\hline $\begin{array}{c}\text { Magnification } \\
\text { Value }\end{array}$ & Linear Method & $\begin{array}{c}\text { Exponential } \\
\text { method }\end{array}$ & ANN & SEMMicro001 \\
\hline X 100 & 99.19 & 99.99 & 98.61 & 100 \\
X200 & 100.02 & 99.99 & 99.60 & 100 \\
X 300 & 49.93 & 49.99 & 49.63 & 50 \\
X 400 & 50.10 & 49.99 & 49.80 & 50 \\
X 500 & 50.20 & 49.99 & 49.90 & 50 \\
X 600 & 19.91 & 19.99 & 19.79 & 20 \\
X 700 & 19.95 & 19.99 & 19.83 & 20 \\
X 800 & 19.99 & 19.99 & 19.87 & 20 \\
X 900 & 20.02 & 19.99 & 19.90 & 20 \\
\hline
\end{tabular}

Table (4): A comparison between linear, exponential and ANN model with valid software at magnifications from X 1000 to $X 9000$

\begin{tabular}{ccccc}
\hline $\begin{array}{c}\text { Magnification } \\
\text { Value }\end{array}$ & $\begin{array}{c}\text { Linear } \\
\text { Method }\end{array}$ & $\begin{array}{c}\text { Exponential } \\
\text { method }\end{array}$ & ANN & SEMMicro001 \\
\hline X 1000 & 9.92 & 10.00 & 9.86 & 10 \\
X2000 & 10.02 & 10.00 & 9.96 & 10 \\
X 3000 & 4.99 & 5.00 & 4.96 & 5 \\
X 4000 & 5.01 & 5.00 & 4.98 & 5 \\
X 5000 & 5.02 & 5.00 & 4.99 & 5
\end{tabular}




\begin{tabular}{lllll} 
X 6000 & 1.99 & 2.00 & 1.98 & 2 \\
X 7000 & 2.00 & 2.00 & 1.98 & 2 \\
X 8000 & 2.00 & 2.00 & 1.99 & 2 \\
X 9000 & 2.00 & 2.00 & 1.99 & 2 \\
\hline
\end{tabular}

Table (5): A comparison between linear, exponential and ANN model with valid software at magnifications from X 10000 to

\begin{tabular}{|c|c|c|c|c|}
\hline $\begin{array}{c}\text { Magnification } \\
\text { Value }\end{array}$ & Linear Method & $\begin{array}{c}\text { Exponential } \\
\text { method }\end{array}$ & ANN & SEMMicro001 \\
\hline X 10000 & 0.99 & 1.00 & 0.99 & 1 \\
\hline X20000 & 1.00 & 1.00 & 1 & 1 \\
\hline X 30000 & 0.5 & 0.5 & 0.5 & 0.5 \\
\hline X 40000 & 0.5 & 0.5 & 0.5 & 0.5 \\
\hline X 50000 & 0.5 & 0.5 & 0.5 & 0.5 \\
\hline X 60000 & 0.2 & 0.2 & 0.2 & 0.2 \\
\hline X 70000 & 0.2 & 0.2 & 0.2 & 0.2 \\
\hline X 80000 & 0.2 & 0.2 & 0.2 & 0.2 \\
\hline X 90000 & 0.2 & 0.2 & 0.2 & 0.2 \\
\hline
\end{tabular}

Table (6): A comparison between linear, exponential and ANN model with valid software at magnifications from X 100000 to $\mathrm{X} 300000$

\begin{tabular}{ccccc}
\hline $\begin{array}{c}\text { Magnification } \\
\text { Value }\end{array}$ & Linear Method & Exponential method & ANN & SEMMicro001 \\
\hline X 100000 & 0.1 & 0.1 & 0.1 & 0.1 \\
X200000 & 0.1 & 0.1 & 0.1 & 0.1 \\
X300000 & 0.05 & 0.05 & 0.05 & 0.05 \\
\hline
\end{tabular}

\section{Conclusion}

By increasing the magnification, the value of microbar within each image decreases. The developed mathematical model can describe the relation between pixel values and micro size at different magnifications. The approximation that was applied on the model led to increase the validity and accuracy of the developed model. However, the model merits are limited to certain magnifications power. The linear, exponential and ANN approximation lie within the accepted accuracy. The performance of the trained data in artificial neural network was $6 \times 10^{-6}$.

One of the applied approximations (exponential) may exceed the others in accuracy. This is remarkable only at a higher micro size, but it becomes slighter at smaller micro size. The model is of a great benefit for nuclear security and safeguards, especially when dealing with environmental samples and routine safeguards inspections.

\section{References}

1. Beane, R. J. (2004). Using the scanning electron microscope for discovery based learning in undergraduate courses. Journal of Geoscience Education, 52(3), 250-253.

2. Shaban, S.E., Akl, Z.F., Zidan, W.I., \& Elshereafy, E. (2015). An optimized 
method for characterization and verification of nano sized uranium and thorium particles for safeguards purposes. Journal of Chemistry and Materials Research, Vol.7 No.10.

3. Aharinejad, S. H., \& Lametschwandtner, A. (1992). Fundamentals of scanning electron microscopy. In Microvascular Corrosion Casting in Scanning Electron Microscopy (pp. 44-51). Springer, Vienna.

4. Togawa, K., Baba, H., Onoe, K., Inagaki, T., Shintake, T., \& Matsumoto, H. (2004). $\mathrm{CeB}_{6}$ electron gun for the soft X-ray fel project at spring-8. Nuclear Instruments and Methods in Physics Research Section A: Accelerators, Spectrometers, Detectors and Associated Equipment, 528(1-2), 312315.

5. Goldstein, J. I., Newbury, D. E., Echlin, P., Joy, D. C., Fiori, C., \& Lifshin, E. (1984). Scanning electron microscopy and X-ray microanalysis Plenum Press. New York, London.
6. Goldman I.N., (1994). Environmental sampling and analysis" ,IAEA-SM-33197, International Nuclear Safeguards proceedings,Vol.1.

7. Spector, Leonard S. Nuclear ambitions: the spread of nuclear weapons 1989-1990. Routledge, 2019.

8. Kips, R., Leenaers, A., Tamborini, G., Betti, M., Van den Berghe, S., Wellum, R., \& Taylor, P. (2007). Characterization of uranium particles produced by hydrolysis of $\mathrm{UF}_{6}$ using SEM and SIMS. Microscopy and Microanalysis, 13(3), 156-164.

9. Shaban, S. E., El-Tayebany, R. A., \& Hazzaa, M. H. (2019). Construction of a developed software for nuclear forensics of micro particles. Journal of Radiation Research and Applied Sciences, 12(1), 101-105. 